East African Medical Journal Vol. 87 No. 6 June 2010

INCIDENCE OF POST DURAL PUNCTURE HEADACHE FOLLOWING CAESAREAN SECTION UNDER SPINAL ANAESTHESIA AT THE AGA KHAN UNIVERSITY HOSPITAL, NAIROBI

E. Gisore, MBChB, MMed (Anaest.), Instructor, V. Mung'ayi, MBChB, MMed (Anaest.), FICM, Assistant Professor and T.Sharif, MBChB, MMed (Anaest.), G.Dip. (Neuro Sc.) MMed, Pain Management (Sydney), Senior Instructor, Department of Anaesthesia, Aga Khan University Hospital, Nairobi, P.O. Box 30270 - 00100, Nairobi, Kenya

Request for reprints to: Prof. V. Mung'ayi, Department of Anaesthesia, Aga Khan University Hospital, Nairobi, P.O. Box 30270 - 00100, Nairobi, Kenya

\title{
INCIDENCE OF POST DURAL PUNCTURE HEADACHE FOLLOWING CAESAREAN SECTION UNDER SPINAL ANAESTHESIA AT THE AGA KHAN UNIVERSITY HOSPITAL, NAIROBI
}

\author{
E. GISORE, V. MUNG'AYI and T. SHARIF
}

\begin{abstract}
Background: Post dural puncture headache can be a debilitating complication of subarachnoid anaesthesia for a new mother. Successful management of post dural puncture headache requires adherence to clear policies and protocols with close follow up of patients by an experienced obstetric anaesthetist.

Objectives: To examine the cumulative incidence and severity of post dural puncture headache in obstetric patients who consented for spinal anaesthesia for Caesarean section.

Design: Prospective cohort study.

Subjects: All women who underwent Caesarean sections under spinal anaesthesia and fulfilled the inclusion criteria.

Setting: Aga Khan University Hospital, a teaching and referral hospital in Nairobi, Kenya.

Results: The overall cumulative incidence of post dural puncture headache was found to be $20.35 \%$ but was significantly higher in patients in whom the quincke type of needle was used than in those whose spinal anaesthetics were administered using the pencil point needle $(24.2 \%$ and $4.5 \%$ respectively: $\mathrm{p}=0.042)$.

Conclusions: The incidence of post dural puncture headache can be significantly reduced in the obstetric population at the Aga Khan University Hospital if the pencil point spinal needle was to be routinely used. We recommend that the Quincke needles should not be used in the obstetric population at the Aga Khan University Hospital.
\end{abstract}

\section{INTRODUCTION}

Spinal anaesthesia is an inexpensive, safe, reliable and rapidly effective method of providing regional anaesthesia for Caesarean section $(1,2)$. Epidural anaesthesia, which is quite popular in developed countries $(3,4)$, may not be readily available in the developing countries because of the cost and technical skills needed toestablish an epidural service. Spinal anaesthesia would therefore appear to be appropriately suited for developing countries with their meagre resources and dearth of highly skilled anaesthetists (5). Unfortunately, spinal anaesthesia is sometimes complicated by the occurrence of a post dural puncture headache (PDPHA), especially in the obstetric patient $(5,6)$.

A Post Dural Puncture Headache (PDPHA) is usually described as the headache (HA) after lumbar puncture (LP). A definition of PDPHA conforming to the International Headache Society criteria is a bilateral HA that develops within seven days after an LP and disappears within 14 days after the LP. The headache occurs or worsens within 15 minutes of assuming the upright position and disappears or improves within 30 minutes of resuming the recumbent position (7). The risk factors for PDPHA are well described but most of the published literature is from developed countries (3-6).

At the Aga Khan University Hospital in Nairobi, Kenya, a substantial proportion of Caesarean sections are done under general anaesthesia for various reasons. Spinal anaesthesia is just beginning to gain popularity in our obstetric unit. When we perform spinal blocks, the type and gauge of needle used depends on the availability of the needle and the preference of the individual anaesthetist.

Personal communication with anaesthetists in the Aga Khan University Hospital confirms that the 
most commonly used spinal needle in our obstetric unit is the 25-gauge Quincke needle and most anaesthetists claim to have a very low incidence of PDPHA in their patients. These are, however, unpublished reports. There is a paucity of published reports about spinal anaesthesia and PDPHA from countries in East Africa. This study was designed to examine prospectively the cumulative incidence and severity of PDPHA in parturients undergoing Caesarean sections under spinal anaesthesia at the Aga Khan University Hospital, Nairobi.

This hospital has a busy obstetric unit with an annual delivery rate of about two thousand and like in most hospital settings, Caesarean section is one of the most common surgical procedures carried out in the hospital. An audit done at the Aga Khan University Hospital (AKUH), showed Caesarean section rates of $22-62 \%$ in 2007 . In the same study, the overall Caesarean section rate was $20.4 \%$ in $1996,25.9 \%$ in 2001 and $38.1 \%$ in 2004 . The rate among patients managed by private obstetricians was $27.1 \%$ in 1996 , $30.8 \%$ in 2001 and $41.7 \%$ in 2004 . Whilst among general patients, it was $14.7 \%, 21.5 \%$ and $34.5 \%$ respectively (8). The hospital records reveal a general anaesthesia rate for Caesarean delivery to be $65 \%$ compared to the spinal anaesthesia rate which stands at 35\% (13).

According to the Literature, the incidence of PDPHA varies between 5-30\% $(9,10)$. The work of Tourtellotte et al. (11), demonstrated the large variability in the frequency of PDPHA in different settings and in different series and the apparent ability to reduce this frequency based on uncontrolled reports.

The averagefrequency that they reported in their monograph has been replicated or even exceeded in more recent experience e. g 37\% in the series by Kuntz et al. (12), and thus PDPHA is not a problem of the past.

The incidence of PDPHA in Kenyan obstetrical patientsisunknown.Diversity of patientdemographics and differences in anaesthetic administration technique make it difficult to predict with certainty by extrapolation of the studies done elsewhere. In the Aga khan University Hospital, the incidence of PDPHA had not been investigated. This study was to investigate the incidence of PDPHA following Caesarean section in the AKUH, Nairobi.

\section{MATERIALS AND METHODS}

This prospective cohort study was approved by the Aga Khan University Research and Ethics committee on 20th January 2009. The data collection was conducted between January 2009 and A pril 2009 both months inclusive.

All pregnant women who underwent Caesarean delivery, both elective and emergency, under spinal anaesthesia during this period were included in the study after obtaining an informed consent from each patient. All patients were free of the known contraindications to spinal anaesthesia such as bleeding diasthesis, uncorrected hypotension and local infection.

The spinal anaesthetics were administered by the anaesthetists on duty who included permanent employees of the hospital, private practitioners and anaesthetists in training. These anaesthetists were blinded to the study. Independent trained research assistants were assigned to fill a questionnaire that included the following information: In-Patient Number, Patient age, Urgency of Caesarean section, anaesthetist level of experience, Pre-loading done, Needle type, Needle gauge, Number of attempts (number of needle insertions before cerebrospinal fluid emerged), Position and duration time of the procedure from positioning the patient to delivery of the drug. An attempt was defined as starting with the introduction of the spinal needle and ending with the removal of the stylet with anticipation of cerebrospinal fluid flush back. The principal investigator was excluded from administering spinal anaesthetics during the study period.

The principal investigator then made patient follow up twelve hours later and filled a post procedure follow up sheet which included the following:Presence of headache by direct questioning about the classic signs, its onset, its severity and any associated symptoms as well as the need for and response to therapy for the headache.

The severity of the PDPHA was assessed using a Visual Analogue Scale (V AS) extending from 0-10; 0 $=$ no headache, $1-3=$ mild headache, $4-7=$ moderate headache, $>7=$ severe headache.

Patients were also reviewed for presence or absence of neck pains, photophobia, tinnitus, nausea and vomiting. The patients found to have a headache within the first twelve hours had a neurological examination done by the principal investigator and then they were followed up at twenty four, forty eight and seventy two hours later. All patients were given a follow up call at seven days instead of two weeks to minimize on the recall bias.

During the time when we were doing the follow up, we were aggressively involved in instituting the conventionally used pain relief methods. For all mothers under private practitioners who were having post-dural puncture headache, the information was immediately relayed to the anaesthetists in attendance to guide in management of the headache. Only one patient had such severe headache requiring a neurological review.

Statistical methods: Data were collected using structured questionnaires. The collected data were entered into an MS-Excel data base. The data were analysed using STATA10 (StataCorp Texas). A p-value of $<0.05$ was considered statistically significant. 
The study outcomes were presence or absence of headache. The potential risk factors considered were maternal age, parity, anaesthetist level of experience, Pre-loading done, Needle type, Needle gauge, Number of attempts, Position and duration time of the procedure from positioning the patient to delivery of the drug.

The association between headache and various factors (Anaesthetist level of experience, Preloading done, Needle type, Needle gauge, Number of attempts, position and duration time of the procedure from positioning the patient to delivery of the drug) were assessed using a Fishers exact test.

\section{RESULTS}

Atotal of onehundred and thirteen womenundergoing Caesarean section under spinal anaesthesia were recruited into the study after obtaining an informed consent. The mean age was $30.5,95 \% \mathrm{CI}(29.7,31.4)$ years. The range was 19 to 40 years. The mode parity was two $(39.8 \%)$ with a range $=0-9$.

There were a total of seventy emergency $(62 \%)$ and 43 elective (38\%) Caesarean sections. Spinal anaesthesia was performed with a 20-gauge needle in one patient $(0.9 \%), 22$ gauge needle in thirty two $(28.3 \%)$ and 25 gauge in eighty patients $(70.8 \%)$.

Out of forty patients who received spinal anaesthetics by the registrars, eleven of them developed PDPHA (27.5\% ) and out of fifty three patients who had the same anaesthetic administered by a consultant, eight of them, hence $15.1 \%$ developed the PDPHA. However, the association between level of experience of anaesthetist and occurrence of PDPHA was not statistically significant in this study $\mathrm{p}=0.322$. In this study, $12.5 \%$ of women who received more than 1500 millilitres (mls) of fluid developed PDPHA. 19.5\% of those who received between 1000 to $1499 \mathrm{mls}$ of fluid developed PDPHA and 27.2\% of those women who received between half a litre of fluid and 999 mls developed PDPHA. However, this study was not able to demonstrate statistical significance in the reduction in the occurrence of headache between the patients who were pre-loaded and those who were co-loaded, $\mathrm{p}=0.643$.

Twenty three out of 113 patients reported headache that fulfilled the PDPHA criteria. Of the total number of patients with headache, three (13\%) classified their headache as mild ( $<3$ on the VAS). Nine $(39 \%)$ classified their headache as severe (VAS >7) and eleven patients (48\%) classified their headache as moderate ( VAS OF 4-7). Headaches responded to simple analgesics and oral fluids in all patients. None of the patients required an epidural blood patch or prolonged enforced bed rest. One patient required a neurological review but responded well on conservative management.
In this study only $4.5 \%$ of the twenty two mothers whohad the spinal anaesthetic administered using the pencil point pencan needle developed the PDPHA. Compared to their counterparts who had the spinal anaesthetic administered using the quincke, cutting needle, the incidence of PDPHA was $24.2 \%$. This association was found to be statistically significant, $\mathrm{P}=0.042$. The gauge of the spinal needle used was not found to be statistically significant in this study.

\section{DISCUSSION}

Post dural puncture headache is the third most common reason for litigation in obstetric anaesthesia as shown in the data obtained from the American Society of Anaesthesiologists ( ASA)' s closed claims analysis project. The care provider has the responsibility to undertake appropriate measures to predict and prevent the occurrence of debilitating headache.

Post dural puncture headache is an unfortunate complication of spinal anaesthesia. It occurs with a higher incidence in obstetric patients, $(14,15)$ especially when large bore cutting (Quincke) needles are used.

Previous studies have shown a positive correlation between the size and configuration of the hole in the dura and the incidence of PDPHA (16). Two factors determine the size of the whole made in the dura sac: needle size and needle tip configuration.

In this study, the incidence of PDPHA at the Aga Khan University Hospital is $20.35 \%$ which falls between the $5-30 \%$ reported rates. To the best of our knowledge, this is the first report of obstetric spinal anaesthesia and PDPHA in Kenya, where Quincke needles are used by most anaesthetists.

Out of forty patients who received spinal anaesthetics by the registrars, eleven of them developed PDPHA $(27.5 \%)$ and out of fifty three patients who had the same anaesthetic administered by a consultant, eight of them, hence $15.1 \%$ developed the PDPHA. However, the association between level of experience of anaesthetist and occurrence of PDPHA was not statistically significant in this study $\mathrm{P}=0.322$.

In this study, $12.5 \%$ of women who received more than 1500 millilitres (mls) of fluid developed PDPHA. $19.5 \%$ of those who received between 1000 to 1499 mls of fluid developed PDPHA and $27.2 \%$ of those women who received between half a litre of fluid and 999 mls developed PDPHA. However, this study was not able to demonstrate statistical significance in the reduction in the occurrence of headache between the patients who were pre-loaded and those who were co loaded, $\mathrm{p}=0.643$.

In this study only $4.5 \%$ of the twenty two mothers whohad the spinal anaesthetic administered using the pencil point pencan needle developed the PDPHA. 
Compared to their counterparts who had the spinal anaesthetic administered using the quincke, cutting needle, the incidence of PDPHA was $24.2 \%$. This association was found to be statistically significant, $\mathrm{P}=0.042$. The gauge of the spinal needle used was not found to be statistically significant in this study.

\section{RECOMMENDATIONS}

The incidence of post dural puncture headache is within the range of $5-30 \%$ as reported in the studies shown in the literature review. From this study, it is shown that there is an association of the incidence with the type of spinal needle used being lesser with the pencil point needle, $p=0.042$. Other causal association factors shown in other studies like needle gauge and pre-loading were not demonstrated in this study. There is need for further studies with larger sample sizes to demonstrate the impact of the other causal contributing factors.

Quincke needle should be avoided in obstetric patients due to the unacceptably high incidence of PDPHA associated with their use. Additionally, because spinal anaesthesia for Caesarean section is only just beginning to gain popularity in our obstetric unit, it is prudent to avoid anything that would jeopardise patient confidence and informed consent for future use of spinal anaesthesia.

Pencil-point needles are more expensive than cutting (Quincke-type) needles and many obstetric units in developing countries may be unable to afford them. However, the advantages of spinal anaesthesia should be weighed against the risk of PDPHA and the risk should be explained to the patient.

\section{ACKNOWLEDGEMENTS}

To the Aga Khan University Hospital, Nairobi, for giving permission to publish this study.

\section{REFERENCES}

1. Michie, A.R., Freeman, R.M., Dutton, D.A. and Howie, H.B.Subarachnoid anaesthesia for elective caesarean section. Anaesthesia. 1988; 43: 96-99.

2. Gogarten, W. and Van Aken, H. A century of regional analgesia in obstetrics. Anesthesia Analgesia. 2000; 91: 773-775.

3. Clyburn, D. Spinal anaesthesia for caesarean, time for re appraisal. Anaesthesia. 2005; 60: 633-635.
4. Shibli, K.U. and Russell, I.F. A survey of anaesthetic techniques used for caesarean section in the UK in 1997. Intern. J. Obstet. Anaest. 2000; 9: 160-167.

5. Nafiu, O.O., Salam, R.A. and Elegbe, E.O. Post dural puncture headache in obstetric patients: experience from a West African teaching hospital. Intern. J. Obstet. Anaest. 2007; 16: 4-7.

6. Lybecker, H., Moller, J.T., May and Nielsen, H.K. Incidence and prediction of post dural puncture headache: a prospective study of 1021 spinal anesthesias. Anesthesia Analgesia. 1990; 70: 389-394.

7. Evans, R.W., Armon, C. and Frohman, E.M. Assessment: Prevention of post-lumbar puncture headaches: Report of the therapeutics and technology assessment subcommittee of the American Academy of Neurology. Neurology. 2000; 55: 909-914.

8. Turnbull,D.K. and Shepherd,D.B.Postdural puncture headache: pathogenesis, prevention and treatment. Brit. J. Anaest. 2003; 91: 718-729.

9. Raskin, N.H. Lumbar puncture headache: a review. J. Head and Face Pain. 1990; 30: 197-200.

10. Cliff,H.S. and Chadwick, M.D. Obstetric anaesthesia closed claims update II. Intern. J. Obstet. Anaest. 1996; 5: 258-263.

11. Cook, T., Bland, L., Mihai, R. and Scott, S. Litigation related to anaesthesia: an analysis of claims against the NHS in England 1995-2007. Anaesthesia. 2009; 64: 706-718.

12. Mung'ayi, V., Nekyon, D. and Karuga, R. Knowledge, attitude and use oflabour pain relief methods among women attending antenatal clinic in Nairobi. East Afr. Med. J. 2008; 85: 438-441.

13. Wanyonyi, S., Sequeira, T. and Obura, T. Caesarean section rates and perinatal outcome at the Aga Khan University Hospital, Nairobi. East Afr. Med. J. 2007; 83: 651.

14. Foster, P. ACTH treatment for post-lumbar puncture headache. Brit. J. Anaest. 1994; 73: 429.

15. Yucel, A., Ozyalcin, S. and Talu, O.K. Intravenous administration of caffeine sodium benzoate for postdural puncture headache. Regional Anest. Pain Med. 1999; 24:51.

16. Stevens, R. and Jorgennsen, N. Successful treatment of dural puncture headache with epidural saline infusion after failure of epidural blood patch. Acta Anaesthesiologica Scandinavica. 1988; 32: 429-431. 